\title{
Efeito da Estratégia Saúde da Família na redução de internações por doenças crônicas não transmissíveis
}

\author{
Effect of the Family Health Strategy in reducing hospitalizations for chronic non-communicable diseases \\ Efecto de la Estrategia de Salud Familiar en la reducción de hospitalizaciones por enfermedades crónicas no \\ transmisibles
}

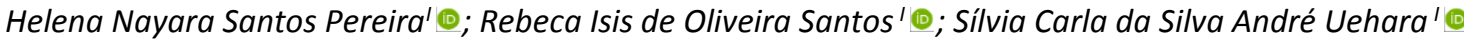 \\ 'Universidade Federal de São Carlos, São Carlos, SP, Brasil
}

\begin{abstract}
RESUMO
Objetivo: analisar o perfil de internações por Doenças Crônicas não Transmissíveis (DCNT) e sua relação com a cobertura da Estratégia Saúde da Família (ESF). Método: estudo descritivo, exploratório e de abordagem quantitativa, realizado em São Carlos-SP, no período de 2016 a 2018. As informações foram coletadas no sistema e-SUS e nas fichas de Autorizações de Internação Hospitalar. Os dados foram analisados por meio da estatística descritiva. A pesquisa foi aprovada pelo Comitê de Ética em Pesquisa da Universidade Federal de São Carlos. Resultados: destaca-se que as taxas de internação por angina, insuficiência cardíaca, diabetes e hipertensão diminuíram, sobretudo entre 2017 e 2018, quando houve ampliação da cobertura de ESF. Conclusão: a expansão da ESF foi determinante para reduzir as internações por DCNT; contudo, faz-se necessário ampliar os serviços prestados aos usuários, e reavaliar as estratégias de cuidado, especialmente aquelas direcionadas aos idosos e indivíduos acometidos por doenças cerebrovasculares e pulmonares.
\end{abstract}

Descritores: Atenção Primária à Saúde; Estratégia Saúde da Família; Doenças não Transmissíveis; Prevenção Primária.

\section{ABSTRACT}

Objective: to analyze the profile of hospitalizations for Chronic non-Communicable Diseases (NCDs) and their relationship with coverage by the Family Health Strategy (FHS). Method: this exploratory, quantitative, descriptive study was conducted from 2016 to 2018 at 21 FHS units in São Carlos, São Paulo State, Brazil. Information was collected from the e-SUS system and Hospitalization Authorization forms and data were analyzed using descriptive statistics. The study was approved by the research ethics committee of São Carlos Federal University. Results: hospitalization rates for angina, heart failure, diabetes, and hypertension were found to have decreased between 2017 and 2018, when FHS coverage expanded. Conclusion: expansion of the FHS was crucial to reduce NCD admissions. However, the services provided to users need to be expanded, and care strategies, especially those directed to the elderly and individuals suffering from cerebrovascular and pulmonary diseases, need to be reassessed and intensified.

Descriptors: Primary Health Care; Family Health Strategy; Noncommunicable Diseases; Primary Prevention.

\section{RESUMEN}

Objetivo: analizar el perfil de las hospitalizaciones por Enfermedades Crónicas no Transmisibles (ENT) y su relación con la cobertura de la Estrategia de Salud de la Familia (ESF). Método: este estudio exploratorio, cuantitativo y descriptivo se realizó de 2016 a 2018 en 21 unidades de la ESF en São Carlos, Estado de São Paulo, Brazil. Se recopiló información del sistema e-SUS y de los formularios de Autorización de Hospitalización y se analizaron los datos mediante estadística descriptiva. El estudio fue aprobado por el comité de ética en investigación de la Universidad Federal de São Carlos. Resultados: se encontró que las tasas de hospitalización por angina, insuficiencia cardíaca, diabetes e hipertensión disminuyeron entre 2017 y 2018, cuando se expandió la cobertura de la ESF. Conclusión: la expansión de la ESF fue crucial para reducir los ingresos por ENT. Sin embargo, es necesario ampliar los servicios que se brindan a los usuarios y reevaluar e intensificar las estrategias de atención, especialmente las dirigidas a las personas mayores y a las personas que padecen enfermedades cerebrovasculares y pulmonares.

Descriptores: Atenación Primaria de Salud; Estrategia de Salud Familiar; Enfermedades no Transmisibles; Prevención Primaria.

\section{INTRODUÇÃO}

As Doenças Crônicas Não Transmissíveis (DCNT), caracterizadas por serem multifatoriais e de longa duração, são responsáveis por 41 milhões de mortes anuais no mundo, tendo impacto principalmente em países em desenvolvimento. Entre essas enfermidades, o grupo constituído por Diabetes Mellitus (DM), Hipertensão Arterial Sistêmica (HAS), doenças cardiovasculares, neoplasias e doenças pulmonares é responsável por mais de $80 \%$ das mortes ${ }^{1-3}$.

A Estratégia Saúde da Família (ESF), implantada no Brasil com vistas a remodelação da Atenção Primária à Saúde (APS), desempenha papel fundamental na redução da incidência das DCNT. A ESF implementa um modelo de assistência

Agradecimento ao Programa Institucional de Bolsas de Iniciação Científica do Conselho Nacional de Desenvolvimento Científico e Tecnológico (CNPq). 
orientado para a prevenção e promoção da saúde, com a participação da comunidade e guiado por uma equipe multidisciplinar ${ }^{2,4}$.

Este estudo se justifica por reconhecer a efetividade e capacidade da ESF na redução de Internações por Condições Sensíveis à Atenção Primária (ICSAP), além de reforçar a necessidade de investimentos na APS para superar esses desafios, mesmo diante de crises econômicas, uma vez que se constitui em um dos principais pilares de sustentação do Sistema Único de Saúde (SUS). Assim, objetivou-se avaliar o perfil de internações hospitalares por DCNT em São CarlosSP e sua relação com a cobertura de ESF.

\section{REFERENCIAL TEÓRICO}

As DCNT respondem por cerca de $80 \%$ das mortes registradas nas Américas. Essas doenças poderiam ser evitadas por meio da adoção de hábitos alimentares saudáveis, prática de atividades físicas, redução da ingestão de bebidas alcoólicas e abandono do tabaco. As DCNT são decorrentes dos processos de transição demográfica, nutricional e epidemiológica, agravados por fatores inerentes ao indivíduo como sexo, idade e características genéticas ${ }^{5,6}$.

Entre a população adulta brasileira, a presença de ao menos uma DCNT está associada à maior frequência de utilização dos serviços de saúde e internações ${ }^{7}$. O diagnóstico precoce e o tratamento adequado são essenciais para reduzir a incidência de mortes prematuras decorrentes das complicações das DCNT 5 .

As ações implementadas em âmbito nacional tentam minimizar os impactos das DCNT, em especial as atividades realizadas pela ESF, que oferece atenção integral e contínua ao usuário, destacando-se o acompanhamento domiciliar, atendimentos coletivos, além de exames para diagnóstico precoce e detecção de possíveis complicações ${ }^{8-10}$.

A evolução desses indicadores de saúde, sobretudo a diminuição da morbimortalidade por diferentes tipos de enfermidades, tem motivado a realização de estudos desde a implementação da ESF. Quando comparada ao modelo clássico de cuidado à saúde, a ESF alcança resultados mais satisfatórios e possui potencial para reduzir a morbimortalidade por DCNT entre os usuários da APS ${ }^{11}$.

No período de 2001 a 2016, podem ser observadas evidências de redução da taxa de Internações por Condições Sensíveis à Atenção Primária (ICSAP), caracterizadas como um grupo de causas de internações e diagnósticos, conforme estabelecido na Décima Revisão da Classificação Internacional de Doenças (CID-10), e listadas na Portaria no 221/2008 do Ministério da Saúde. Essa redução, que alcançou 45\%, foi resultado da ampliação do acesso a medicamentos e melhorias no acompanhamento e diagnóstico das doenças, proporcionados pela ESF $^{12,13}$

A lista de ICSAP abrange 74 classes de enfermidades, agrupadas em 18 categorias, das quais fazem parte as DCNT. O comportamento das taxas de ICSAP, sobretudo nas áreas de abrangência das ESF, representa um importante indicador da qualidade dos cuidados que são prestados aos usuários, podendo fomentar as futuras decisões de gestores de saúde para a necessidade de reformulação de estratégias ${ }^{13,14}$.

A inclusão das DCNT na lista de ICSAP relaciona-se ao fato de essas doenças representarem um preocupante problema de saúde pública, contribuindo para a elevação dos custos associados ao seu tratamento tanto por parte dos indivíduos quanto do sistema público de saúde. Essas enfermidades apresentam expressiva participação nas taxas de mortalidade. No Brasil, do total de óbitos de indivíduos na faixa etária dos 30 aos 69 anos, 56\% foram ocasionados por alguma DCNT; no município de São Paulo mais de 70\% de todas as mortes são ocasionadas por essas doenças ${ }^{3,6,7,15}$.

Apesar de já existirem pesquisas sobre o tema em questão, destaca-se que os estudos apresentam dados que atestam a importância do atendimento humanizado prestado na APS e a associação entre a ampliação da cobertura de ESF e as taxas de internação por DCNT ${ }^{16-20}$. Este estudo apresenta uma análise temporal, abrangendo um período de três anos, da evolução das DCNT, visando relacionar esses dados à cobertura da ESF; e, indiretamente, avaliar a contribuição das ações desenvolvidas atualmente no contexto da APS para modificação do perfil de morbidade.

\section{MÉTODO}

Trata-se de um estudo descritivo e exploratório, observacional e de abordagem quantitativa, utilizando dados secundários em séries temporais de São Carlos, São Paulo, que possui uma população estimada de 238.958 habitantes e uma cobertura de ESF aproximada de 75.900 habitantes $^{21}$. Na área da saúde, em 2018, a APS do município era composta por 12 Unidades Básicas de Saúde (UBS) e 21 ESF.

Os dados foram coletados no e-SUS e nas fichas de Autorizações de Internação Hospitalar na Secretaria de Saúde, referente ao período de 2016 a 2018. Para a categorização das internações como ICSAP utilizou-se a Lista de Condições Sensíveis à Atenção Primária que consta na Portaria no 221/2008 ${ }^{13}$. As variáveis coletadas das AIH foram: CID; idade; bairro; logradouro e CEP. 
Neste estudo foi considerada como variável desfecho as condições crônicas relacionadas às taxas de ICSAP. De acordo com a CID-10, as DCNT consideradas condições sensíveis à atenção ambulatorial são: asma, doenças pulmonares, HAS, angina, Insuficiência Cardíaca (IC), doenças cerebrovasculares, DM e epilepsias ${ }^{12}$. Para este estudo foram selecionas: HAS, DM, angina, doenças pulmonares e doenças cerebrovasculares.

Foram coletadas informações referentes ao total de hospitalizações por DCNT em áreas do município em que havia ESF e, também, naquelas não cobertas pela Estratégia. Realizou-se, levantamento da frequência de internações de acordo com cada uma das DCNT preestabelecidas, nos três anos, a fim de verificar se a ampliação da ESF, além de exercer influência na alteração das taxas totais de internação, também modificaria o perfil de ocorrência de DCNT específicas, determinando sua redução.

Foram coletados dados sobre a faixa etária dos pacientes internados em decorrência das DCNT, no intuito de identificar padrões que justificariam a necessidade de reformulação e/ou intensificação das estratégias direcionadas a determinados grupos populacionais. Os dados foram duplamente digitados em planilha do Excel e agrupadas de acordo com a CID 10 e analisados pela estatística descritiva.

A pesquisa foi aprovada pelo Comitê de Ética em Pesquisa (CEP) da instituição, sob o número do Certificado de Apresentação para Apreciação Ética (CAAE) 02162018.5.0000.5504.

\section{RESULTADOS E DISCUSSÃO}

No período de 2016 a 2018, foram registradas 4.025 internações por DCNT em São Carlos; 32,15\% (1.294) ocorreram em 2016; 36,30\% (1.461) em 2017; e, em 2018, 31,55\% (1.270). Verifica-se que no período de 2016 a 2017 houve uma taxa de crescimento de 12,9\% e, em 2018, um decréscimo de 13,07\% em relação ao ano anterior.

Esse incremento no número de internações por DCNT não necessariamente atesta que a população esteja adoecendo com maior frequência ao longo dos anos, mas pode ser reflexo da gradativa ampliação da oferta e acesso aos serviços de saúde, bem como da otimização dos procedimentos diagnósticos sobretudo nas consultas preventivas. Essa afirmação é corroborada por uma pesquisa que analisou a evolução da oferta de estabelecimentos e recursos de saúde no país nas últimas três décadas, demonstrando que houve um aumento significativo no número de estabelecimentos, de 21.532 em 1981 para 129.544 em $2017^{22}$.

Já a diminuição no número das internações, observada em 2018, pode estar associada à ampliação da cobertura da ESF no município, que em dezembro de 2017 era de 28,31\%, atendendo uma população estimada de 69.000 pessoas; em 2018, houve um aumento da cobertura da ESF, que passou a ser de 30,84\%, atendendo uma população de 75.900 pessoas $^{23}$. O aumento da cobertura de ESF beneficia a população mais vulnerável e contribui para tornar a APS mais eficiente e evitar que usuários sejam encaminhados para outros níveis de atenção à saúde sem necessidade.

Em relação às causas de internações por DCNT, no ano de 2016 predominaram àquelas decorrentes de angina, representando $25 \%$ (324) dos casos, e IC que concentrou 25\% (324) dos casos. Ainda, 10,4\% (134) das internações ocorreram em consequência de DM e 2,7\% (35) de HAS (Figura 1).

Doenças do Aparelho Circulatório (DAC) lideram as estatísticas de causa de morte há décadas no mundo ${ }^{24-25}$. No Brasil, em 2011, essas doenças representaram as principais causas de óbitos, correspondendo a $28,6 \%{ }^{25}$. $A$ multiplicidade de fatores de risco associados pode ser determinante na predominância das internações por doenças associadas ao sistema cardiovascular, pois aumenta a demanda por monitoramento e cuidados contínuos, dificultando a adesão do paciente ao tratamento.

O padrão observado em 2016 manteve-se em 2017, ano em que os registros de internações por DCNT foram decorrentes, em sua maioria, de angina (401 casos, 27,45\% das internações) e IC (345 casos, 23,61\% das internações). Entre as menores taxas de internação mantiveram-se as taxas ocasionadas por DM com 142 (9,27\%) e HAS com 32 (2,19\%) internações; essas taxas se apresentaram ainda menores em 2018, quando foram registradas 72 (5,7\%) internações por DM e apenas 16 (1,3\%) por HAS. Os referidos dados podem ser observados na Figura 1.

A frequência de internações por DM e HAS deve-se aos esforços que vêm sendo empreendidos sobretudo a partir de 2001 com a introdução do Plano de Reorganização da Atenção a HAS e DM na APS. Esse Plano tem como propósito assegurar e facilitar o acesso dos indivíduos acometidos por essas patologias aos serviços de saúde, que devem investir na prevenção e no diagnóstico precoce ${ }^{26}$. Ainda, deve-se considerar que parte dessas internações não necessariamente tem como causa primária a HAS ou DM, mas resultam de suas complicações como Acidente Vascular Encefálico (AVE), Infarto Agudo do Miocárdio (IAM), doença renal crônica, problemas circulatórios, renais e oftalmológicos.

Estudo sobre os fatores associados à busca por atendimento de emergência e internação por portadores de HAS e DM evidenciou que 20,7\% (113 pacientes) das consultas emergenciais e 6\% (33 pacientes) das internações hospitalares 
foram decorrentes de alguma complicação dessas enfermidades ${ }^{27}$. Ressalta-se, que indivíduos com HAS apresentam complicações com maior frequência, sobretudo IAM e AVE, quando comparados aos indivíduos diabéticos ${ }^{28}$.

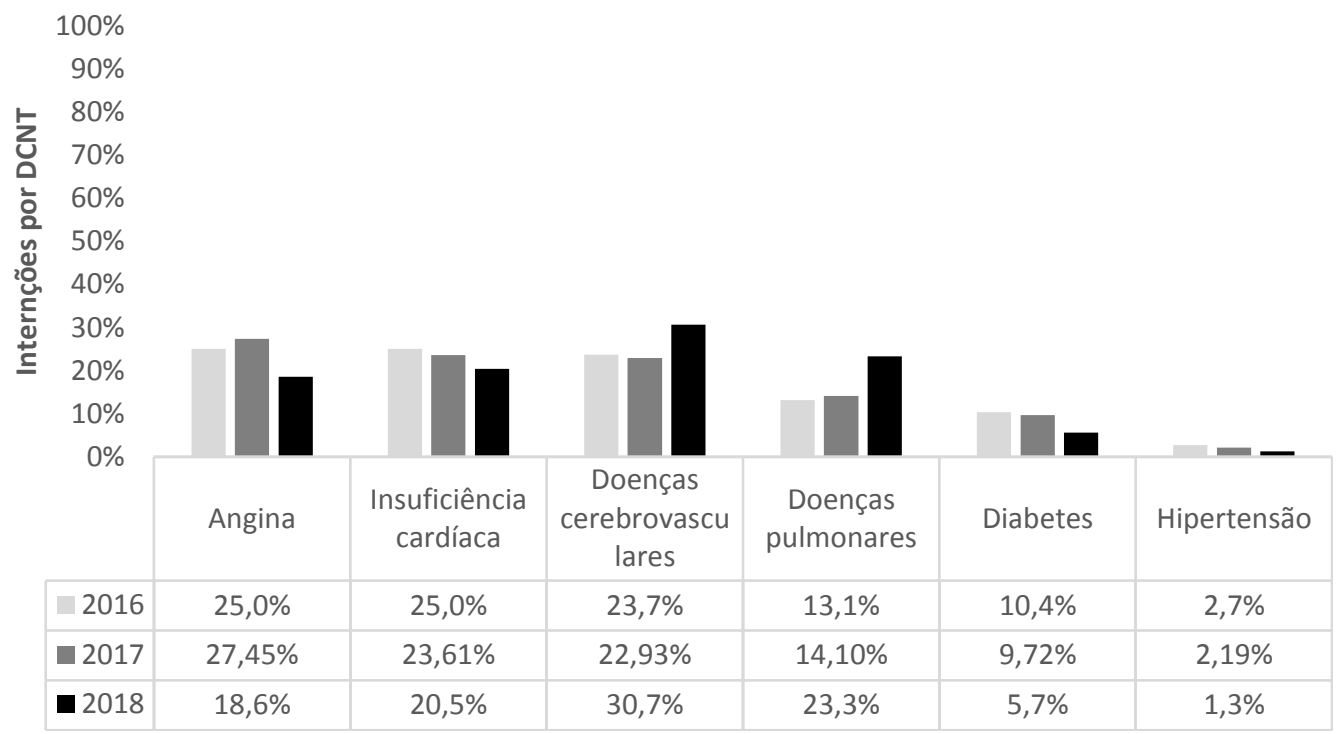

Figura 1: Perfil de internações por DCNT no período de 2016 a 2018 no município de São Carlos, São Paulo, Brasil, 2019.

Diante desse cenário, as ações direcionadas para a educação em saúde desenvolvidas na APS e o aumento da cobertura de ESF são fundamentais para a queda nas taxas de internação por DM e HAS, observada em diferentes regiões brasileiras, como evidenciam estudos realizados em Goiás, Paraná, Minas Gerais, Pernambuco e Espírito Santo ${ }^{14,16-20}$.

Em 2018, 30,7\% (390) das internações por DCNT em São Carlos foram decorrentes das doenças cerebrovasculares. Estudo sobre as tendências da doença cerebrovascular no Brasil no período de 1990 a 2015 constatou aumento no número de casos e óbitos ${ }^{29}$. Em relação à mortalidade por essas doenças, foi verificado uma redução de óbitos entre os idosos nas últimas décadas na maioria das regiões brasileiras ${ }^{30}$. A redução da mortalidade pode estar relacionado às mudanças de estilos de vida e controle dos fatores de risco para as doenças cerebrovascular como tabagismo, dislipidemias, hipertensão arterial, alimentação inadequada, diabetes e sedentarismo ${ }^{31,32}$. O acesso ampliado aos serviços de saúde, em especial a expansão da APS e as melhorias nas condições socioeconômicas da população contribuem para esclarecer essa diminuição da mortalidade por doenças cerebrovasculares na população ${ }^{33,34}$.

Entretanto, mesmo diante da redução da mortalidade nas regiões mais desenvolvidas, as doenças cerebrovasculares, ainda são a principal causa de morte no país ${ }^{33}$. Assim, as ações para a prevenção e controle dos fatores de risco para morbimortalidade dessas doenças, bem como a identificação precoce dos sinais e sintomas devem continuar sendo uma prioridade de saúde pública ${ }^{34}$.

A APS deve fortalecer o desenvolvimento de ações que visem minimizar a influência dos fatores de risco modificáveis como sobrepeso, obesidade, sedentarismo e tabagismo. Estudo que analisou fatores de risco e complicações em pessoas diabéticas e hipertensas mostrou que sobrepeso e obesidade constituem, conjuntamente, um dos fatores de risco mais prevalentes entre esses indivíduos, especialmente aqueles que apresentam HAS isoladamente ou em associação ao DM; ainda, 44,01\% dos participantes eram sedentários ${ }^{28}$.

O sedentarismo representa uma das principais causas do excesso de peso, resultando em alterações metabólicas que elevam o risco de desenvolvimento de DCNT, é razoável inferir que as atividades que visam incentivar a prática de exercícios físicos entre os usuários da APS assumem fundamental importância no contexto da ESF.

Neste estudo, destaca-se a elevação nas taxas de internação decorrentes de doenças pulmonares, responsáveis por 23,3\% (296) em 2018 (Figura 1). De acordo com a Organização Pan Americana da Saúde as doenças pulmonares estão incluídas entre as dez principais causas de morte no mundo, destacando-se Doença Pulmonar Obstrutiva Crônica (DPOC), infecções das vias aéreas inferiores e cânceres de pulmão, traqueia e brônquios, que foram responsáveis por $54 \%$ das 56,9 milhões de mortes que ocorreram no mundo em $2016^{35}$.

As mortes por doenças respiratórias podem estar associadas a fatores como o tabagismo e à exposição passiva à fumaça do cigarro. A pesquisa do sistema de Vigilância de Fatores de Risco e Proteção para Doenças Crônicas por 
Inquérito Telefônico (VIGITEL), em 2018, apresentou que 12,5\% da população adulta de São Paulo são fumantes, além de $6,8 \%$ e $6,7 \%$ identificados como fumantes passivos no domicílio e no trabalho, respectivamente ${ }^{36}$. As pessoas tabagistas com alguma DCNT relacionada ao tabaco devem ser assistidas em todos os níveis de assistência, especialmente no que se refere às estratégias direcionadas para as mudanças comportamentais ${ }^{37,38}$.

Outro aspecto que contribui para a elevação dos índices de doenças respiratórias agudas e crônicas refere-se à exposição a altos níveis de poluição do ar, que ultrapassam o limite máximo recomendado pelas diretrizes da qualidade do ar da Organização Mundial da Saúde (OMS) em diversos países, incluindo o Brasil $^{5}$.

As ICSAP relacionadas às pneumonias bacterianas reduziram em $4 \%$ em crianças menores de um ano, tal redução pode estar associada, em partes, às estratégias preventivas desenvolvidas na APS 39

A piora da qualidade do ar decorre de inúmeros processos que envolvem a ação do homem, como as queimadas e a queima de combustíveis nas indústrias e automóveis, afetam sobretudo a saúde das crianças, idosos e indivíduos que apresentam histórico de doenças respiratórias. As crianças compreendem a população mais vulnerável, sendo que cerca de $93 \%$ estão expostas a níveis deletérios de poluição atmosférica. No Brasil, diferente do que ocorre em outros países, a poluição do ar não é tratada como um fator de risco para as DCNT, apesar das evidências que comprovam suas implicações negativas na qualidade de vida e o consequente aumento de gastos relacionados às consultas médicas, internações e uso de medicamentos entre todas as faixas etárias ${ }^{40}$.

Ressalta-se que neste estudo, foi considerada a faixa etária definida pelo Estatuto da Criança e do Adolescente (ECA), Lei 8.069 de 1990, que considera criança a pessoa até 12 anos de idade incompletos e define a adolescência como a faixa etária entre 12 e 18 anos de idade; e pelo Estatuto do Idoso, que considera pessoa idosa aquela com idade igual ou superior a 60 anos. Para critério de comparação entre as fases do ciclo vital adotou-se também a divisão jovem adulto (19 a 24 anos); adulto (25 a 40 anos) e adulto de meia idade (40 a 60 anos) ${ }^{40}$.

No que diz respeito as internações por faixa etária, verifica-se que estão concentradas na faixa etária de pessoas acima de 60 anos. Em 2016, 60,74\% (786) das internações em decorrência de alguma DCNT eram de idosos; porém, houve uma redução nas taxas de internação nos anos subsequentes, sendo registradas 851 ( $58,24 \%$ do total) de internações de idosos em 2017 e 730 (57,48\% do total) em 2018 (Tabela 1). Este resultado é condizente com os dados de uma pesquisa que analisou as tendências das DCNT no Brasil no período de 2002 a 2012, em que se observou maiores taxas de internação entre as faixas etárias mais elevadas ${ }^{41}$.

As internações por IC concentraram pessoas com mais de 60 anos acometidas, sendo 76,5\% (248) em 2016, 75,1\% (259) em 2017 e 78,8\% (205) em 2018; das internações decorrentes de angina, 58,3\% (189) foram registradas entre idosos no ano de 2016, enquanto no ano de 2017 e 2018 as proporções foram de 57,9\% (232) e 60,6\% (143), respectivamente; em relação às doenças cerebrovasculares, $69,4 \%$ das internações ocorreram na faixa etária acima dos 60 anos, proporção que atingiu 68,1\% (228) em 2017 e 70,3\% (274) em 2018 (Tabela 1). Esses achados, além de refletirem a maior vulnerabilidade e suscetibilidade a determinadas condições de saúde, características dessa faixa etária, evidenciam que a escassez de recursos financeiros, perda da memória e solidão, podem ter impacto negativo sobre a qualidade da alimentação e a ingestão de medicamentos.

Destaca-se o maior percentual de internações por doenças pulmonares entre crianças de 0 a 11 anos de idade, seguido pela população na faixa etária acima dos 60 anos, padrão que difere do perfil das demais DCNT avaliadas, como apresentado na Tabela 1.

A asma é considerada uma doença pulmonar de elevada incidência em crianças, afetando 235 milhões de pessoas em todo o mundo ${ }^{42}$. Também, considera-se a participação de outras doenças pulmonares de impacto mundial, como a pneumonia, uma das principais causas de morte em crianças menores de cinco anos; DPOC, responsável por 3 milhões de mortes anuais; além de tuberculose e câncer de pulmão que ocasionam, respectivamente 1,4 milhão e 1,6 milhão de óbitos todos os anos ${ }^{42}$.

Análise sobre as ICSAP em Santa Catarina identificou que as doenças pulmonares correspondem à terceira causa de hospitalização, responsável por $16,9 \%$ das internações entre crianças de até 5 anos. Esses resultados não necessariamente atestam incapacidade de resolução dos problemas de saúde da população na APS, mas indicam lacunas na operacionalização que, se sanadas, contribuirão para reduzir as taxas de internação por causas evitáveis ${ }^{43}$. As lacunas podem ter diversas origens, desde escassez de pessoal capacitado até a falta de itens básicos para a realização das consultas, medicamentos e aparelhos essenciais para o diagnóstico, carências decorrentes dos constantes cortes na verba destinada ao SUS.

Portanto, o crescimento da cobertura e acesso à APS não implica, necessariamente, em melhora na qualidade dos serviços prestados à população, havendo, necessidade de investimentos em contratação e qualificação dos profissionais, aquisição de equipamentos e manutenção das unidades. 
TABELA 1: Perfil das internações por DCNT de acordo com a faixa etária no período de 2016 a 2018 no município de São Carlos, São Paulo, Brasil, 2019.

\begin{tabular}{|c|c|c|c|c|c|c|c|}
\hline \multirow{2}{*}{ CAUSA } & & \multicolumn{2}{|c|}{2016} & \multicolumn{2}{|c|}{2017} & \multicolumn{2}{|c|}{2018} \\
\hline & & $\mathrm{n}$ & $\%$ & $\mathrm{n}$ & $\%$ & $\mathrm{n}$ & $\%$ \\
\hline \multirow[t]{6}{*}{ Hipertensão } & 19-24 anos & 1 & 2,8 & 1 & 3,1 & 1 & 6,25 \\
\hline & $25-40$ anos & 4 & 11,4 & 1 & 12,5 & 2 & 12,5 \\
\hline & $41-50$ anos & 3 & 8,5 & 4 & 3,1 & 2 & 12,5 \\
\hline & $51-60$ anos & 5 & 14,3 & 1 & 3,1 & 1 & 6,25 \\
\hline & $>60$ anos & 22 & 63,0 & 1 & 78,2 & 10 & 62,5 \\
\hline & Total & 35 & 100 & 25 & 100 & 16 & 100 \\
\hline \multirow[t]{8}{*}{ Diabetes } & 0-11 anos & 6 & 4,5 & 5 & 3,5 & 3 & 4,2 \\
\hline & $12-18$ anos & 12 & 8,9 & 12 & 8,4 & 4 & 5,6 \\
\hline & 19-24 anos & 3 & 2,2 & 7 & 5,0 & 4 & 5,6 \\
\hline & $25-40$ anos & 17 & 12,7 & 12 & 8,4 & 8 & 11,0 \\
\hline & $41-50$ anos & 12 & 8,9 & 7 & 5,0 & 11 & 15,3 \\
\hline & $51-60$ anos & 27 & 20,2 & 35 & 24,6 & 15 & 20,8 \\
\hline & $>60$ anos & 57 & 42,6 & 64 & 45,1 & 27 & 37,5 \\
\hline & Total & 134 & 100 & 142 & 100 & 72 & 100 \\
\hline \multirow[t]{6}{*}{ Angina } & 19-24 anos & 1 & 0,3 & 0 & 0,0 & 0 & 0,0 \\
\hline & $25-40$ anos & 6 & 1,9 & 10 & 2,5 & 4 & 1,7 \\
\hline & $41-50$ anos & 34 & 10,5 & 29 & 7,2 & 22 & 9,3 \\
\hline & $51-60$ anos & 94 & 29,0 & 130 & 32,4 & 67 & 28,4 \\
\hline & $>60$ anos & 189 & 58,3 & 232 & 57,9 & 143 & 60,6 \\
\hline & Total & 324 & 100 & 401 & 100 & 236 & 100 \\
\hline \multirow[t]{8}{*}{ Insuficiência cardíaca } & 0-11 anos & 1 & 0,3 & 5 & 1,4 & 5 & 1,9 \\
\hline & $12-18$ anos & 0 & 0,0 & 0 & 0,0 & 1 & 0,4 \\
\hline & 19-24 anos & 0 & 0,0 & 0 & 0,0 & 2 & 0,8 \\
\hline & $25-40$ anos & 6 & 1,9 & 5 & 1,4 & 4 & 1,5 \\
\hline & $41-50$ anos & 16 & 5,0 & 23 & 6,7 & 8 & 3,1 \\
\hline & $51-60$ anos & 53 & 16,3 & 53 & 15,4 & 35 & 13,5 \\
\hline & $>60$ anos & 248 & 76,5 & 259 & 75,1 & 205 & 78,8 \\
\hline & Total & 324 & 100 & 345 & 100 & 260 & 100 \\
\hline \multirow[t]{8}{*}{ Doenças cerebrovasculares } & 0-11 anos & 1 & 0,32 & 0 & 0,0 & 1 & 0,26 \\
\hline & $12-18$ anos & 0 & 0,0 & 1 & 0,3 & 0 & 0,0 \\
\hline & 19-24 anos & 0 & 0,0 & 4 & 1,2 & 12 & 3,1 \\
\hline & $25-40$ anos & 11 & 3,6 & 19 & 5,7 & 12 & 3,1 \\
\hline & $41-50$ anos & 23 & 7,5 & 35 & 10,4 & 36 & 9,2 \\
\hline & $51-60$ anos & 59 & 19,2 & 48 & 14,3 & 55 & 14,1 \\
\hline & $>60$ anos & 213 & 69,4 & 228 & 68,1 & 274 & 70,3 \\
\hline & Total & 307 & 100 & 335 & 100 & 390 & 100 \\
\hline \multirow[t]{8}{*}{ Doenças pulmonares } & 0-11 anos & 90 & 53,0 & 146 & 70,9 & 196 & 66,2 \\
\hline & $12-18$ anos & 0 & 0,0 & 0 & 0,0 & 1 & 0,3 \\
\hline & 19-24 anos & 0 & 0,0 & 1 & 0,5 & 0 & 0 \\
\hline & $25-40$ anos & 2 & 1,2 & 1 & 0,5 & 4 & 1,4 \\
\hline & $41-50$ anos & 6 & 3,5 & 4 & 1,94 & 6 & 2,0 \\
\hline & $51-60$ anos & 15 & 8,8 & 11 & 5,3 & 18 & 6,1 \\
\hline & $>60$ anos & 57 & 33,5 & 43 & 20,9 & 71 & 24,0 \\
\hline & Total & 170 & 100 & 206 & 100 & 296 & 100 \\
\hline
\end{tabular}

As internações por DCNT foram maiores entre os usuários residentes em áreas da cidade que não possuíam ESF. Em 2016, 16,3\% (211) das internações por DCNT eram de usuários oriundos de áreas com cobertura de ESF; e, 83,7\% (1.083) das internações eram de pessoas residentes em áreas sem cobertura pela ESF. Em 2017, o percentual de internações por DCNT na área com cobertura da ESF aumentou para 20,6\% (301); enquanto 79,4\% (1.160) das internações eram de pessoas residentes em áreas sem cobertura. Já em 2018, houve um decréscimo nas internações por DCNT de pessoas residentes em área de cobertura em relação ao ano anterior; 18,7\% (238) das internações 
foram registradas entre os indivíduos residentes na área de cobertura, enquanto nas áreas sem cobertura foi de 81,3\% (1.032), como apresentado na Figura 2.

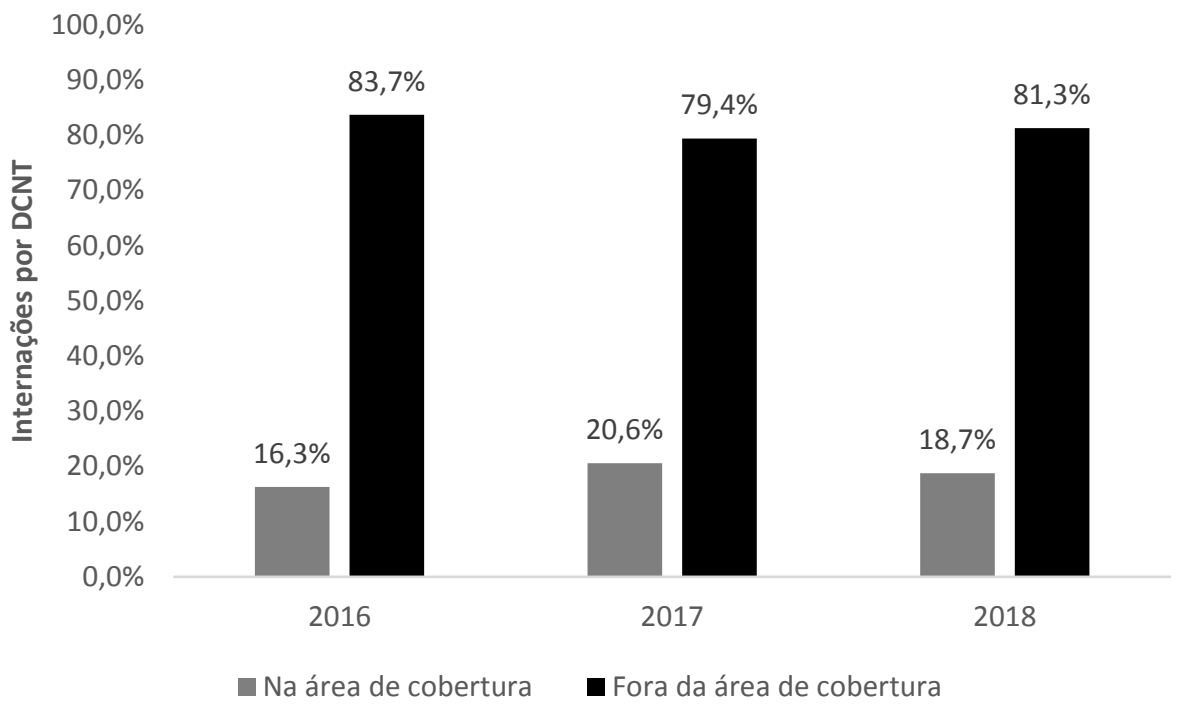

FIGURA 2: Percentual de internações por DCNT no período de 2016 a 2018 em áreas cobertas e não cobertas pela ESF no município de São Carlos, São Paulo, 2019.

O investimento em ações estratégicas, como a ampliação da cobertura de ESF, além de ocasionar um decréscimo nas taxas de internações por DCNT, e garantir a disponibilidade de leitos hospitalares para os casos de maior gravidade, reduz as despesas com saúde pública, permitindo o remanejamento dos recursos dentro do próprio sistema ${ }^{44}$.

As ESF são os principais serviços que promovem a participação dos usuários no sistema público de saúde por meio das ações de prevenção e promoção da saúde, além de fomentar a adesão aos tratamentos; reduz, os custos com deslocamentos até centros especializados de saúde e pode ser uma importante estratégia para a modificação de indicadores de mortalidade geral e infantil. Considera-se, a necessidade de ampliação das ESF levando-se em conta não somente as necessidades de saúde, mas também fatores socioeconômicos das populações.

Não obstante a relevância dos achados, este estudo teve como limitação a falta de preenchimento de alguns campos da ficha de AlH.

\section{CONCLUSÃO}

Observou-se redução nas taxas de internação decorrentes de angina, IC, DM e HAS, mais acentuada no período de 2017 a 2018, quando houve ampliação da cobertura de ESF em São Carlos, que beneficia sobretudo a população mais vulnerável.

As ICSAP estudadas concentraram-se, em sua maioria, na faixa etária acima de 60 anos, evidenciando uma maior vulnerabilidade da população idosa e pode, ainda, ser considerado um reflexo do longo período de convivência com DCNT e comorbidades associadas. Entretanto, a faixa etária de 0 aos 11 concentrou o maior percentual de internações por doenças pulmonares.

Considerando que o percentual de internações decorrentes das DCNT avaliadas foi consideravelmente mais elevado entre os usuários em áreas não cobertas pela ESF, conclui-se que os investimentos na APS, sobretudo no sentido de garantir o acesso de um maior número de pessoas à ESF e evitar complicações, são determinantes para a redução nas taxas dessas internações.

\section{REFERÊNCIAS}

1. World Health Organization. Noncommunicable diseases [Internet]. 2018. [cited in 2020 Feb 26]. Available from: https://www.who.int/news-room/fact-sheets/detail/noncommunicable-diseases

2. Pan American Health Organization/World Health Organization. Innovative Care for Chronic Conditions: Organizing and Delivering High Quality Care for Chronic Noncommunicable Diseases in the Americas [Internet]. [cited 2020 Feb 26]. Available from: https://www.paho.org/hq/index.php?option=com_content\&view=article\&id=8500:2013-innovative-delivering-highquality-care-chronic-noncommunicable-diseases\&ltemid=1353\&lang=en 
3. Ministério da Saúde (Br). Secretaria de Vigilância em Saúde. Departamento de Vigilância de Doenças e Agravos não Transmissíveis e Promoção da Saúde. Coordenação Geral de Vigilância de Doenças e Agravos não Transmissíveis e Promoção da Saúde. Nota informativa [Internet]. 2018. [cited 2020 Mar 12]. Available from: https://portalarquivos2.saude.gov.br/images/pdf/2018/novembro/23/Nota-informativan-final.pdf

4. Ministério da Saúde (Br). Estratégia Saúde da Família (ESF) [Internet]. [cited 2020 Mar 12]. Available from: https://www.saude.gov.br/acoes-e-programas/saude-da-familia/implantacao-da-estrategia

5. Pan American Health Organization/World Health Organization. Noncommunicable Disease Mortality and Risk Factor Prevalence in the Americas [Internet]. 2019. Available from:

https://iris.paho.org/bitstream/handle/10665.2/51696/PAHONMH19014_eng.pdf?sequence=9\&isAllowed=y

6. Prefeitura Municipal de São Paulo. Secretaria Municipal de Saúde. Doenças Crônicas não Transmissíveis - DCNT [Internet]. 2019. [cited 2020 Mar 12]. Available from:

https://www.prefeitura.sp.gov.br/cidade/secretarias/saude/vigilancia_em_saude/doencas_e_agravos/index.php?p=6099

7. Malta DC, Bernal RTI, Lima MG, Araújo SSC, Silva MMA, Freitas MIF, et al. Noncommunicable diseases and the use of health services: Analysis of the National Health Survey in Brazil. Rev. Saude Publica. 2017 [cited 2020 Mar 12]; 51:1-10. DOI: https://doi.org/10.1590/s1518-8787.2017051000090

8. Becker RM, Heidemann ITSB, Meirelles BHS, Costa MFBNA, Antonini FO, Durand MK. Nursing care practices for people with Chronic Noncommunicable Diseases. Rev. Bras. Enferm. [Internet]. 2018 [cited 2020 Mar 4]; 71(6):2643-71. DOI: http://dx.doi.org/10.1590/0034-7167-2017-0799

9. Ministério da Saúde (Br). Estratégia Saúde da Família (ESF) [Internet]. [cited 2020 Mar 4]. Available from: https://www.saude.gov.br/acoes-e-programas/saude-da-familia/sobre-o-programa

10. Medina MG, Aquino R, Vilasbôas ALQ, Mota E, Júnior EPP, Luz LA, et al. Health promotion and chronic disease prevention: what are Family Health teams doing? Saúde Debate [Internet]. 2014 [cited 2020 Mar 26]; 38:69-82. Available from: http://www.scielo.br/pdf/sdeb/v38nspe/0103-1104-sdeb-38-spe-0069.pdf

11. Jardim LV, Navarro D. Contribution of the control FHS chronic noncommunicable diseases. J. Heal. Sci. Inst. [Internet]. 2017. [cited 2020 Mar 26]; 35(2):122-8. Available from: https://www.unip.br/presencial/comunicacao/publicacoes/ics/edicoes/2017/02_abr-jun/V35_n2_2017_p122a126.pdf

12. Pinto LF, Giovanella L. The Family Health Strategy: expanding access and reducing hospitalizations due to ambulatory care sensitive conditions (ACSC). Cien. Saude Colet. [Internet]. 2018. [cited 2020 Mar 26]; 23(6):1903-14. Available from: http://www.scielo.br/scielo.php?script=sci_arttext\&pid=S1413-81232018000601903\&lng=pt\&tlng=pt

13. Ministério da Saúde (Br), Secretaria de Atenção à Saúde. Portaria n²21, de 17 de abril de 2008 [Internet]. [cited 2020 Mar 26]. Available from: http://bvsms.saude.gov.br/bvs/saudelegis/sas/2008/prt0221_17_04_2008.html

14. Maia LG, Silva LA, Guimarães RA, Pelazza BB, Pereira ACS, Rezende WL, et al. Hospitalizations due to primary care sensitive conditions: an ecological study. Rev. Saude Publica [Internet]. 2019. [cited 2020 Mar 9]; 53(2):1-10. DOI: https://doi.org/10.11606/S1518-8787.2019053000403

15. Ministério da Saúde (Br). Vigilância de Doenças Crônicas não Transmissíveis (DCNT) [Internet]. [cited 2020 Mar 26]. Available from: https://www.saude.gov.br/vigilancia-em-saude/vigilancia-de-doencas-cronicas-nao-transmissiveis-dcnt

16. Pazó RG, Frauches DDO, Molina MDCB, Cade NV. Overview of hospitalizations for ambulatory care sensitive conditions in Espirito Santo, Brazil, 2000 to 2014. Rev. Bras. Med. Família e Comunidade. 2017; 12(39):1-12. DOI: https://doi.org/10.5712/rbmfc12(39)1546

17. Ribeiro TH, Magri CL, Santos AL. Hospitalization due to diabetes mellitus in adults and its relationship with first aid expansion in Paraná, Brazil. Saúde e Pesqui [Internet]. 2019. [cited 2020 Mar 09]; 12(2):232-331. Available from: http://docs.bvsalud.org/biblioref/2019/09/1016522/10_7039-thaisnara_port_norm_ing.pdf

18. Fernandes TF, Pereira MI, Fernandes VBL, Grilo LEM, Rocha SR, Maciel AG. Morbimortality by diabetes in the municipality of Montes Claros - MG. Ver. Adm. Saúde [Internet]. 2018 [cited 2020 Mar 09]; 18(71). Available from: http://www.cqh.org.br/ojs2.4.8/index.php/ras/article/view/100/143

19. Diniz RO. Estudo sobre a evolução das internações hospitalares por hipertensão essencial (primária), no município de Medina, Minas Gerais: uma patologia sensível ao cuidado primário no período de 2000 a 2010 [Internet]. 2011 [cited 2020 Mar 09]. Available from: https://ares.unasus.gov.br/acervo/html/ARES/4657/1/3017.pdf

20. Mendonça SS, Albuquerque EC. Profile of hospital admissions for primary care sensitive conditions in Pernambuco State, Brazil, 2008-2012. Epidemiol e Serviços Saúde. 2014 [cited 2020 Mar 09]; 23(3):463-74. DOI: http://dx.doi.org/10.5123/S167949742014000300009

21. Instituto Brasileiro de Geografia e Estatística. IBGE Cidades: São Carlos. [cited 2020 Mar 09]. Available from: http://www.cidades.ibge.gov.br/xtras/perfil.php?lang=\&codmun=354890\&search=sao-paulo|sao-carlos

22. Viacava F, Oliveira RAD, Carvalho CC, Laguardia J, Bellido JG. SUS: supply, access to and use of health services over the last 30 years. Cien. Saude Colet. 2018 [cited 2020 Mar 09]; 23(6):1751-62. DOI: https://doi.org/10.1590/1413-81232018236.06022018

23. Ministério da Saúde (Br). e-Gestor AB [Internet]. 2017. [cited 2020 Mar 09]. Available from: https://egestorab.saude.gov.br/paginas/acessoPublico/relatorios/relHistoricoCoberturaAB.xhtml

24. Silva MVM, Oliveira VS, Pinto PMA, Razia PFS, Caixeta ACL, Aquino ÉC, et al. Trends of hospitalizations for ambulatory caresensitive cardiovascular conditions in the municipality of Senador Canedo, Goiás, Brazil, 2001-2016. Epidemiol. e Serv. saude Rev. do Sist. Unico Saude do Bras. 2019 [cited 2020 Mar 09]; 28(1):e2018110. DOI: https://doi.org/10.5123/s167949742019000100018

25. Soares GP, Brum JD, Oliveira GM, Klein CH, Souza e Silva NA. Evolution of socioeconomic indicators and cardiovascular mortality 
in three Brazilian states. Arq Bras Cardiol. 2013;100(2):147-56. [cited 2020 Aug 17]. Available from: https://www.scielo.br/scielo.php?script=sci_arttext\&pid=S0066-782X2013000200007

26. Ministério da Saúde (Br). Departamento de Átenção Básica. Área Técnica de Diabetes e Hipertensão Arteiral. Hipertensão arterial sistêica (HAS) e Diabetes mellitus: protocolo [Internet]. Saúde M da, organizador. Vol. III, Secretaria de Políticas de Saúde. Brasília; 2001. [cited 2020 Mar 09]. 96 p. Available from: http://bvsms.saude.gov.br/bvs/publicacoes/caderno_atencao_basica_n1_p1.pdf

27. Freitas PS, Matta SR, Mendes LVP, Luiza VL, Campos MR. Use of health services and medicines by hypertensive and diabetic patients in the municipality of Rio de Janeiro, Brazil. Cien Saude Colet [Internet]. 2018. [cited 2020 Mar 27]; 23(7):2383-92. DOI: http://dx.doi.org/10.1590/1413-81232018237.21602016

28. Sousa NA , Lima JS, Teixeira TC, Linhares CB, Montes JVL, Marques JVS. Risk factors and complications in diabetic/hypertensive patients registered in the hiperdia. Sanare [Internet]. 2019. [cited 2020 Mar 27]; 18(01):31-9. Available from: https://sanare.emnuvens.com.br/sanare/article/view/1303/684

29. Lotufo PA, Goulart AC, Passos VMA, Satake FM, Souza MFM, França EB, et al. Cerebrovascular Disease in Brazil from 1990 to 2015: Global Burden of Disease 2015. Rev. Bras. Epidemiol. [Internet]. 2017. [cited 2020 Mar 3]; 20(suppl 1):129-41. Available in: http://www.scielo.br/scielo.php?pid=S1415-790X2017000500129\&script=sci_arttext\&tlng=en

30. Guimarães RM, Andrade SSCA, Machado EL, Bahia CA, Oliveira MM, Jacques FVL. Regional differences in cardiovascular mortality transition in Brazil, 1980 to 2012. Rev. Panam. Salud. Pública [Internet]. 2015 [cited 2020 Mar 27]; 37:83-9. Available from: https://scielosp.org/article/rpsp/2015.v37n2/83-89/

31. Yusuf S, Hawken S, Ounpuu S, Dans T, Avezum A, Lanas F, et al. Effect of potentially modifiable risk factors associated with myocardial infarction in 52 countries (the INTERHEART study): case-control study. Lancet [Internet]. 2004 [cited 2020 Mar 27]; 364:937-52. DOI: https://doi.org/10.1016/s0140-6736(04)17018-9

32. Souza MFM, Alencar AP, Malta DC, Moura L, Mansur AP. Serial temporal analysis of ischemic heart disease and stroke death risk in 5 regions of Brazil from 1981 to 2001. Arq. Bras. Cardiol. 2006 [cited 2020 Mar 27]; 87:735-40. Available from: https://www.scielo.br/scielo.php?script=sci_abstract\&pid=S0066-782X2006001900009\&lng=en\&nrm=iso

33. Schmidt MI, Duncan BB, Azevedo e Silva G, Menezes AM, Monteiro CA, Barreto SM, et al. Chronic non communicable diseases in Brazil: burden and current challenges. Lancet [Internet]. 2011 [cited 2020 Mar 27]; 377:1949-61. DOI: https://doi.org/10.1016/s0140-6736(11)60135-9

34. Duncan BB, Chor D, Aquino EML, Bensenor IM, Mill JG, Schmidt MI, et al. Chronic Non-Communicable Diseases in Brazil: priorities for disease management and research. Rev. Saúde Pública [Internet]. 2012 [cited 2020 Mar 27]; 46 Suppl 1:126-34. DOI: https://doi.org/10.1590/S0034-89102012000700017

35. Organização Pan Americana da Saúde/Organização Mundial da Saúde. 10 principais causas de morte no mundo [Internet]. [cited 2020 Mar 27]. Available from: https://www.paho.org/bra/index.php?option=com_content\&view=article\&id=5638:10principais-causas-de-morte-no-mundo\&Itemid $=0$

36. Ministério da Saúde (Br). Secretaria de Vigilância em Saúde. Departamento de Análise em Saúde e Vigilância de Doenças não Transmissíveis. Vigitel Brasil 2018: vigilância de fatores de risco e proteção para doenças crônicas por inquérito telefônico: estimativas sobre frequência e distribuição sociodemográfica de fatores de risco e proteção para doenças crônicas nas capitais dos 26 estados brasileiros e no Distrito Federal [Internet]. Brasília; 2019. 132 p. [cited 2020 Mar 27]. Available from: https://portalarquivos2.saude.gov.br/images/pdf/2019/julho/25/vigitel-brasil-2018.pdf

37. Corrêa da Silva LC, Araújo AJ, Queiroz ÂM, Sales MD, Castellano MV; Comissão de Tabagismo da SBPT. Smoking control: challenges and achievements. J. Bras. Pneumol. [Internet] 2016 [cited 2020 Mar 27]; 42(4):290-8. Available from: http://www.jornaldepneumologia.com.br/detalhe_artigo.asp?id=2566

38. Schmidt MI, Duncan BB, G, Menezes AM, Monteiro CA, Barreto SM, et al. Chronic non-communicable diseases in Brazil: burden and current challenges. Lancet. 2011;377(9781):1949-61. [cited 2018 Aug 26]. Available from: https://www.thelancet.com/pdfs/journals/lancet/PIIS0140-6736(11)60135-9.pdf

39. Costa LQ, Pinto Junior EP, Silva, MGC da. Time trends in hospitalizations for Ambulatory Care Sensitive Conditions among children under five years old in Ceará, Brazil, 2000-2012. Epidemiol. Serv. Saúde. 2017 [cited 2020 Mar 27]; 26(1):51-60. DOI: https://doi.org/10.5123/s1679-49742017000100006

40. Wassall A. Poluição do ar: a vilã da pós modernidade. Cienc. Cult. [Internet]. 2019 [cited 2020 Mar 27]; 71(1):22-4. Available from: http://cienciaecultura.bvs.br/pdf/cic/v71n1/v71n1a08.pdf

41. Santos MAS, Oliveira MM, Andrade SSCA, Nunes ML, Mallta DC, Moura L. Non-communicable chronic disease hospital morbidity trends in Brazil, 2002-2012. Epidemiol. Serv. Saúde [Internet]. 2015. [cited 2020 Mar 27]; 24(3):389-98. Available from: https://www.scielosp.org/pdf/ress/2015.v24n3/389-398

42. World Health Organization. Chronic respiratory diseases [Internet]. [cited 2020 Mar 9]. Available from: https://www.who.int/health-topics/chronic-respiratory-diseases\#tab=tab_1

43. Mariano TSO, Nedel FB. Hospitalization for ambulatory care sensitive conditions in children under five years old in Santa Catarina state, Brazil, 2012: a descriptive study. Epidemiol. e Serv. saude [Internet]. 2018. [cited 2020 Mar 20];27(3). DOI: https://doi.org/10.5123/S1679-49742018000300006

44. Morimoto T, Costa JSD. Hospitalization for primary care susceptible conditions, health spending and Family Health Strategy: an analysis of trends. Cienc. Saude Colet. [Internet]. 2017 [cited 2020 Mar 9]; 22(3):891-900. Available from:

https://www.scielo.br/scielo.php?pid=S1413-81232017002300891\&script=sci_arttext\&tlng=en 